\title{
Flavour independent Search for Hadronically decaying Higgs Bosons
}

\section{Ivo van Vulpen*}

NIKHEF, Amsterdam, The Netherlands

E-mail: Ivo.van.Vulpen@nikhef.nl

\begin{abstract}
The four LEP collaborations ALEPH, DELPHI, L3 and OPAL have collected around $2.5 \mathrm{fb}^{-1}$ of $\mathrm{e}^{+} \mathrm{e}^{-}$data at centre-of-mass energies ranging from 189 to $209 \mathrm{GeV}$. This note presents the results from a search for neutral Higgs boson production in the processes $\mathrm{e}^{+} \mathrm{e}^{-} \rightarrow \mathrm{hZ}$ and $\mathrm{e}^{+} \mathrm{e}^{-} \rightarrow \mathrm{hA}$ where no assumption is made on the flavour of the hadronic decay products of the Higgs boson. No evidence for a signal is observed and the results are presented as model independent limits on the production cross section for neutral Higgs bosons decaying into hadrons as a function of the Higgs boson mass(es). These limits allow to test predictions from a wide range of (future) models.

Preliminary combined results from the four collaborations are presented in the $\mathrm{hZ}$ channel. In the hA channel results from the OPAL and DELPHI collaboration are presented separately. In the $\mathrm{hZ}$ channel, assuming Higgs boson production with a SM cross section decaying exclusively into hadrons, a limit on the mass of the Higgs boson can be set at $112.9 \mathrm{GeV} / \mathrm{c}^{2}$.
\end{abstract}

International Europhysics Conference on High Energy Physics

July 21st - 27th 2005

Lisboa, Portugal

\footnotetext{
* On behalf of the LEP collaborations ALEPH, DELPHI, L3 and OPAL
} 


\section{Introduction}

As long as bosonic decays are kinematically closed, the Standard Model (SM) Higgs boson decays preferentially into the heaviest fermion pair. In most of the mass range accessible at LEP this leads to the dominance of a Higgs decaying into $b \bar{b}$. In extensions of the SM however the coupling of the Higgs boson to b-quarks might well be suppressed and decay into gluons or light quark pairs might be important. This can for example occur in the Minimal Supersymmetric Standard Model (MSSM) and in general Two Higgs Doublet Models (2HDM). Since the search for the SM Higgs boson strongly relies on the identification of b-quarks to separate a possible signal from the background, SM analyses have a reduced sensitivity to these alternative models.

To cover such scenario's the LEP collaborations have developed analyses that search for hadronically decaying Higgs bosons but that are insensitive to the flavour of the quarks/gluons in the decay. Results from these flavour independent searches can be presented as model independent cross section limits on hadronically decaying Higgs boson(s) as a function of the Higgs boson mass(es). These results can be interpreted in a wide range of (future) models.

This note presents the flavour independent search for neutral Higgs bosons at LEP for the hZ and hA channel. Since no evidence for a signal is observed, the results are presented as limits on production cross sections.

\section{Neutral Higgs boson production at LEP}

For centre-of-mass energies accessible at LEP the $\mathrm{h}$ and A bosons are expected to be produced predominantly through two processes: Higgsstrahlung $\mathrm{e}^{+} \mathrm{e}^{-} \rightarrow \mathrm{hZ}$ and pair-production $\mathrm{e}^{+} \mathrm{e}^{-} \rightarrow$ hA. The Feynman diagrams for both processes are shown in Fig.1.

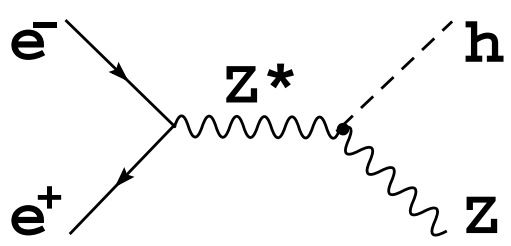

Higgsstrahlung

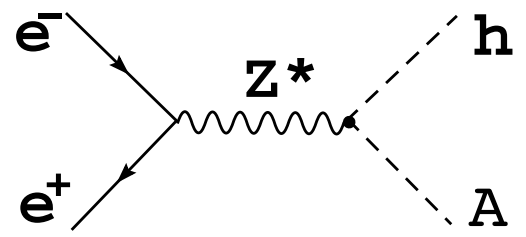

Pair production

Figure 1: Neutral Higgs boson production at LEP.

In the $\mathrm{hZ}$ channel the production rate for any final state can be expressed in terms of the SM hZ cross section, $\sigma_{\mathrm{hZ}}^{\mathrm{SM}}$. Suppression terms coming from branching fractions and possible suppression of the Higgs boson couplings to the $\mathrm{Z}$ boson all factorize and can be written as a generic (model independent) suppression factor $C_{\mathrm{hZ}}^{2}: \sigma_{\mathrm{hZ} \rightarrow \mathrm{Z}+\text { hadrons }}=\sigma_{\mathrm{hZ}}^{\mathrm{SM}} \times C_{\mathrm{hZ}}^{2}$. Also for the pair production process the details of the Higgs sector can be hidden in such a universal reduction factor. In the next two sections the results for each of the two production mechanisms are presented separately as limits on $C_{\mathrm{hZ}}^{2}$ for a range of Higgs boson mass hypotheses. 


\section{Search for $h Z$ production}

In all analyses the Higgs boson is assumed to decay into quark pairs of arbitrary flavour or into gluon pairs. The $\mathrm{hZ}$ final states (search channels) depend therefore on the decay of the $\mathrm{Z}$ boson: the four jet channel $(Z \rightarrow \mathrm{q} \overline{\mathrm{q}} \sim 70 \%)$, the missing energy channel $(\mathrm{Z} \rightarrow v \bar{v} \sim 20 \%)$ and the lepton channels $\left(\mathrm{Z} \rightarrow l^{+} l^{-} \sim 10 \%\right)$. For all search channels the search strategies are similar to those of the SM Higgs boson [1], except that the b-tagging requirements are replaced by more elaborate kinematic selections. The analyses require an object with an invariant mass close to the $m_{\mathrm{Z}}$ and a recoil mass compatible with the Higgs boson mass under investigation.

Even though the quark flavour is not used in the analysis, there are small differences in performance between the various decay products of the Higgs boson, arising from differences in mass resolution and jet structure. In particular, Higgs boson decays into gluon pairs have larger multiplicities, but at the same time coarser dijet mass distribution than decays into light quarks. To allow the results to be presented as genuine flavour independent results for each Higgs boson mass, the sample used for the final evaluation was conservatively chosen as that which gave the weakest expected performance in each channel at that particular mass.

No evidence for a signal was observed and upper limits on the production cross section as a function of the Higgs boson mass were evaluated for each collaboration separately (see references in [2]). The range of Higgs mass hypotheses and data sets used to make the cross section exclusion differ between the collaborations. Although some collaborations go down to very small Higgs boson masses, the results from the four collaborations have only been combined for mass hypotheses ranging from $60 \mathrm{GeV} / \mathrm{c}^{2}$ upwards using the likelihood ratio technique [1, 3]. This LEP combined results is presented in Fig.2.

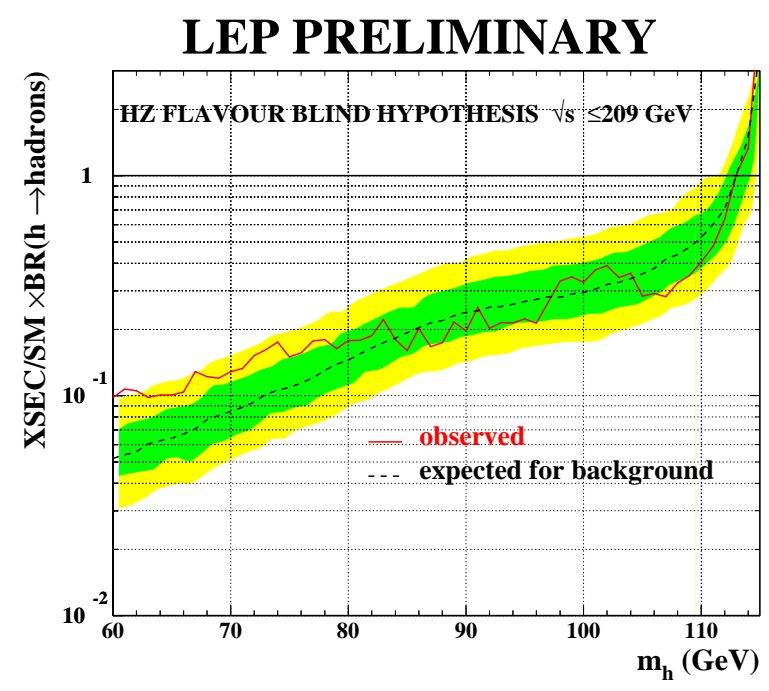

Figure 2: Combined flavour independent LEP observed and expected 95\% CL upper limits on the production cross section as a function of the Higgs boson mass, normalised to the expected Standard Model values, computed assuming BR( $\mathrm{h} \rightarrow$ hadrons $)=1.0$. The computation was done with the likelihood ratio technique [4]. The curves are the observed (solid) and expected median (dashed) excluded ratios, and the bands correspond to $68.3 \%$ and $95 \%$ confi dence intervals from the background-only experiments. 
As an example of the sensitivity of the analysis Table 1 presents the results from the four collaborations as the limit on the mass of a hadronically decaying Higgs boson assuming its cross section is equal to that of the SM Higgs boson. Also the LEP combined limit is presented.

\begin{tabular}{|c|c|c|}
\hline Collaboration & Observed limit $\left(\mathrm{GeV} / \mathrm{c}^{2}\right)$ & Expected Limit $\left(\mathrm{GeV} / \mathrm{c}^{2}\right)$ \\
\hline ALEPH & 109.3 & 108.4 \\
DELPHI & 109.6 & 108.8 \\
L3 & 111.6 & 109.3 \\
OPAL & 109.4 & 108.5 \\
\hline \hline LEP & 112.9 & 113.0 \\
\hline
\end{tabular}

Table 1: Flavour independent observed and expected 95\% CL lower limits on the mass of the Higgs boson, assuming a production cross section equal to that in the $S M$ and $B R(\mathrm{~h} \rightarrow$ hadrons $)=1.0$. The evaluation was performed using the likelihood ratio technique [4]. Systematics were not included.

\section{Search for $h A$ production}

Also in the pair production channel, each Higgs boson is assumed to decay hadronically and to have a negligible width $\left(<1 \mathrm{GeV} / \mathrm{c}^{2}\right)$. The topology of the event depends strongly on $m_{\mathrm{h}}$ and $m_{\mathrm{A}}$ : when both masses are large four well separated jets are expected, whereas if one of the masses is small the two jets from the decay cannot be resolved experimentally. Dedicated analyses are required to cover the full $\left(m_{\mathrm{h}}, m_{\mathrm{A}}\right)$-plane. If kinematically open and allowed by the Higgs boson quantum numbers, cascade decays like $\mathrm{h} \rightarrow$ AA may become dominant. The results presented by the OPAL collaboration have taken this into account.

As was done for the $\mathrm{hZ}$ analysis, to avoid biases in the selection and analysis performance for the different flavours of the hadronic decay products of the Higgs bosons, Monte Carlo samples with different flavour composition have been produced and for each point in the $\left(m_{\mathrm{h}}, m_{\mathrm{A}}\right)$-plane the analysis giving the worst performance has been used in the calculation of the cross section exclusion limit. The SM backgrounds for the fully hadronic hA channel are large and are dominated by $\mathrm{e}^{+} \mathrm{e}^{-} \rightarrow \mathrm{q} \overline{\mathrm{q}}, \mathrm{e}^{+} \mathrm{e}^{-} \rightarrow \mathrm{WW}$ and $\mathrm{e}^{+} \mathrm{e}^{-} \rightarrow$ ZZ events. An additional complication in the analysis of a four jet final state is the jet pairing ambiguity requiring multiple hypotheses to be considered simultaneously.

The OPAL and DELPHI collaborations have observed no evidence for neutral Higgs boson pair production $[5,6]$ and both collaborations have translated their results into upper limits on the production cross section in the $\left(m_{\mathrm{h}}, m_{\mathrm{A}}\right)$-plane as is shown in Figure 3.

The results can be interpreted in a wide range of models where scalar particles are pair produced. At this conference for example the results from a general scan over the 2HDM parameter space was presented by the OPAL collaboration [5] and although in the analysis presented here $\mathrm{CP}$ were assumed to be conserved, analyses have been performed where this assumption was not explicitly made [7] (the so-called MSSM CPX benchmark scenario's). 

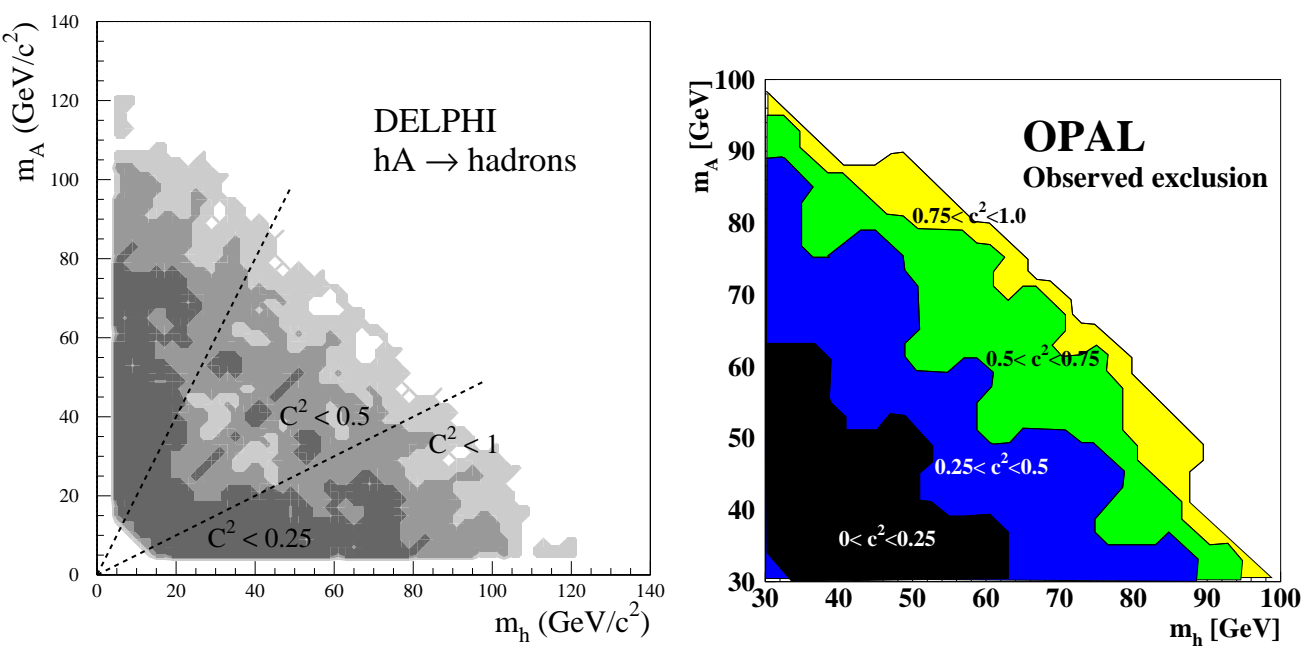

Figure 3: Observed limits on the hA production cross section relative to the reference cross section as a function of the Higgs boson masses for the DELPHI collaboration (left plot) and OPAL collaboration (right plot). Both the $\mathrm{h}$ and $\mathrm{A}$ are assumed to have $100 \%$ branching fractions into hadrons.

\section{Conclusions}

The four LEP collaborations have searched for neutral Higgs boson production and presented the negative search results as upper limits on production cross sections as a function of the Higgs boson mass(es). In the searches presented here the Higgs bosons was assumed to decay into hadrons and no assumptions were made on the flavour of the decay products. These flavour independent cross section limits allow to test a wide range of (future) models against the LEP data.

\section{References}

[1] ALEPH, DELPHI, L3, OPAL Collaborations and the LEP Working Group for Higgs boson searches, Search for the standard model Higgs boson at LEP, Phys. Lett. 3565 (2003), 61-75, hep-ex/0306033.

[2] LEP Working Group for Higgs boson searches, Flavor independent search for hadronically decaying neutral Higgs bosons at LEP, LHWG note 2001-07 (2001), hep-ex/0107034.

[3] A.L. Read, Modifi ed frequentist analysis of search results (The CL(s) method), CERN-Yellow-Report-2000-005 (2000), 81.

[4] T. Junk, Confi dence level computation for combining searches with small statistics, Nucl. Instrum. Meth. A434 (1999), 435-443, hep-ex/9902006.

[5] G. Abbiendi et al., OPAL collaboration, Flavour independent $h^{0} A^{0}$ search and two Higgs doublet model interpretation of neutral Higgs boson searches at LEP, Eur. Phys. J. C40 (2005), 317-332, hep-ex/0408097.

[6] J. Abdallah et al., DELPHI collaboration, Flavour Independent Searches for Hadronically Decaying Neutral Higgs Bosons, Eur. Phys. J. C44 (2005), 147-159.

[7] G. Abbiendi et al., OPAL collaboration, Search for neutral Higgs boson in CP-conserving and CP-violating MSSM scenarios, Eur. Phys. J. C37 (2004), 49-78, hep-ex/0406057. 